\title{
Exploring the Distribution of Blast Resistance Alleles at the Pi2/9 Locus in Major Rice-Producing Areas of China by a Novel Indel Marker
}

\author{
Dagang Tian, ${ }^{1,2}$ Yan Lin, ${ }^{1}$ Ziqiang Chen, ${ }^{1}$ Zaijie Chen, ${ }^{1}$ Fang Yang, ${ }^{2,3}$ Feng Wang, ${ }^{1, \dagger}$ Zonghua Wang, ${ }^{2,4, \dagger}$ and Mo Wang ${ }^{2,3, \dagger}$ \\ ${ }^{1}$ Biotechnology Research Institute, Fujian Key Laboratory of Genetic Engineering for Agriculture, Fujian Academy of Agricul- \\ tural Sciences, Fuzhou, Fujian 350003, China \\ ${ }^{2}$ State Key Laboratory of Ecological Pest Control for Fujian and Taiwan Crops, College of Life Science, Fujian Agriculture and \\ Forestry University, Fuzhou, Fujian 350002, China \\ ${ }^{3}$ Fujian University Key Laboratory for Plant-Microbe Interaction, Fujian Agriculture and Forestry University, Fuzhou, Fujian \\ 350002, China \\ ${ }^{4}$ Institute of Oceanography, Minjiang University, Fuzhou, Fujian 350108, China
}

\begin{abstract}
Rice blast disease caused by the fungus Magnaporthe oryzae damages cereal crops and poses a high risk to rice production around the world. Currently, planting cultivars with resistance $(R)$ genes is still the most environment-friendly approach to control this disease. Effective identification of $R$ genes existing in diverse rice cultivars is important for understanding the distribution of $R$ genes and predicting their contribution to resistance against blast isolates in regional breeding. Here, we developed a new insertion/deletion (InDel) marker, Pigm/2/9InDel, that can differentiate the cloned $R$ genes (Pigm, Pi9, and Pi2/Piz-t) at the Pi2/9 locus. Pigm/2/9InDel combined with the marker Pi2-LRR for $P i 2$ was applied

regions in China. In brief, nine Pigm-containing varieties from Fujian and Guangdong provinces were identified. All of the 62 Pi2-containing varieties were collected from Guangdong, and 60 varieties containing $P i z-t$ were from seven provinces. However, $P i 9$ was not found in any of the Chinese varieties. The newly identified varieties carrying the Pi2/9 alleles were further subjected to inoculation tests with regional blast isolates and field trials. Our results indicate that Pigm and Pi2 alleles have been introgressed for blast resistance breeding mainly in the Fujian and Guangdong region, and $P i 9$ is a valuable blast resistance resource to be introduced into China.
\end{abstract} to determine the distribution of these four $R$ genes among 905 rice varieties, most of which were collected from the major rice-producing
Keywords: blast disease, InDel marker, Pigm, Pi9, Pi2, Piz-t, rice
Rice is a staple food for more than half of the world's population, making it one of the most important cereal crops. Rice blast disease caused by the ascomycete fungus Magnaporthe oryzae (T. T. Hebert) M. E. Barr (syn. Pyricularia oryzae Sacc.) is one of the most devastating diseases threatening rice production worldwide. Breeding rice cultivars with resistance $(R)$ genes has been widely recognized as the most effective and environmentally friendly approach to prevent and control blast disease (Dodds and Rathjen 2010). The genetic structure of the rice blast fungus varies in geographical populations, which leads to regionally specific virulence (Wu et al. 2005). Therefore, identification of $R$ genes existing in diverse rice cultivars is important for estimating the major genetic resources resistant against $M$. oryzae races in rice-producing areas (RoyChowdhury et al. 2012).

More than 100 blast $R$ genes have been identified in rice, of which at least 25 have been cloned (Deng et al. 2017; Liu et al. 2013). The Pi2/9 locus is a cluster of tandem repeats of nucleotide binding site (NBS) leucine-rich repeat (LRR) genes on the short arm of chromosome 6 , and this locus contains at least nine blast $R$ genes. Four

${ }^{\dagger}$ Corresponding authors: F. Wang; wf@fjage.org, Z. H. Wang;

wangzh@fafu.edu.cn, and M. Wang; wangmo108@163.com

Funding: This work was supported by Youngth Program of Fujian Academy of Agricultural Sciences grant YC2019004, National Key R\&D Program of China grant 2016YFD03007007, and Youth Program of National Natural Science Foundation of China grant 31701777.

*The $\boldsymbol{e}$-Xtra logo stands for "electronic extra" and indicates that two supplementary figures and one supplementary table are published online.

The author(s) declare no conflict of interest.

Accepted for publication 10 February 2020.

() 2020 The American Phytopathological Society functional alleles (Pi2, Piz-t, Pi9, and Pigm) of this locus have been cloned, all of which encode NBS-LRR proteins (Deng et al. 2017; $\mathrm{Qu}$ et al. 2006; Zhou et al. 2006). The resistance gene Pi2 was originally introgressed from a resistant indica cultivar into cultivar Co39, and its isogenic line C101A51 displayed broad-spectrum blast resistance (Chen et al. 1996; Mackill and Bonman 1992). Piz- $t$ was cloned based on the $P i 2$ sequence, which is a functional allele of $P i 2$ in rice cultivar Toride 1 (Zhou et al. 2006). Piz-t and Pi2 alleles have high similarity of DNA sequences. $P i 9$ was first introduced into cultivated rice from the wild rice species Oryza minuta, conferring a broader spectrum of blast resistance than that of $P i 2$ in the Philippines (Liu et al. 2002). In 2017, the resistance gene Pigm was identified from Gumei 4, an indigenous Chinese rice variety that has been used as a blast resistance donor for rice breeding in China for $>50$ years (Deng et al. 2017). Because the $P i 2 / 9$ locus contains multiple broad-spectrum $R$ genes, it has been considered as one of the most important loci for resistance against blast disease worldwide (Jiang et al. 2012a; Zhu et al. 2012).

Because new $R$ genes against blast disease are continuously cloned and introgressed in rice cultivars, DNA markers based on the polymorphism of genomic sequence linked to them have been widely developed and used in breeding ( $\mathrm{Fu}$ et al. 2012; Hayashi et al. 2006; Jiang et al. 2012b; Khanna et al. 2015). The utilization of markerassisted selection (MAS) provides an efficient method for directly identifying the introduced $R$ genes controlling resistance phenotypes in population, which overcomes the limitations of conventional breeding. Pita and Pib are the two earliest cloned $R$ genes against blast disease (Bryan et al. 2000; Wang et al. 1999). Development of the DNA markers linked to or from portions of them aids in selecting Pita and Pib rapidly and accurately in rice varieties, providing resistant resources for rice breeding (Jia et al. 2002, 2004; Koide et al. 2010; Liu et al. 2008; Wang et al. 2007; Zhang et al. 2013). DNA markers used for distinguishing polymorphisms belonging to functional and nonfunctional alleles at the Pi2/9 locus have also been developed (Chen et al. 2004; Jiang et al. 2015; Scheuermann and Jia 2016; Wang et al. 2019; Yin et al. 2011). However, with Pigm 
cloned, it still lacks an effective molecular marker to efficiently differentiate multiple functional alleles at the Pi2/9 locus.

In this study, we developed a novel PCR-based insertion/deletion (InDel) marker for distinguishing Pigm, Pi9, Pi2/Piz-t, and nonfunctional alleles named Pigm/2/9InDel and used it to determine the distribution of these four $R$ genes in 905 rice varieties mainly collected from the major rice-producing provinces in China, such as Guangdong, Fujian, Jiangsu, and Zhejiang, and the International Rice Research Institute (IRRI) in the Philippines. Nine, 62, and 60 Chinese varieties carrying Pigm, Pi2, and Piz-t, respectively, were identified, and the only variety carrying $P i 9$ found in this study was IR0436 from the IRRI. Furthermore, inoculation tests with the blast isolates from Guangdong and Fujian showed that Pigm, Pi2, and $P i 9$ are the effective $R$ genes in this area.

\section{Materials and Methods}

Plant materials and $M$. oryzae isolates. Rice blast $R$ gene donors C101A51 (carrying Pi2) and cultivar 75-1-127 (carrying Pi9) were provided by Dr. Guo-Liang Wang (The Ohio State University), and Gumei 4 (carrying Pigm), Toride 1 (carrying Piz-t), and the 905 rice varieties were stored at the Fujian Provincial Key Laboratory of Genetic Engineering for Agriculture, Fujian Academy of Agricultural Sciences, Fuzhou, China. The M. oryzae isolates RB9, RB6, RB16, RB18, CHE86061, CHNOS60-2-3, KJ201, CHL2110, and CHL768 were provided by Dr. Guodong Lu (Fujian Agriculture and Forestry University) and Dr. Qinhua Pan (South China Agricultural University). FJ501-3 and FJ86 were isolated from naturally infected rice plants in a Fujian field.

Evaluation of disease reaction. The $M$. oryzae isolates were grown on oatmeal agar plates in the dark for approximately 5 to 7 days at $28^{\circ} \mathrm{C}$, and then, they were transferred to a culture room under light for approximately 5 to 7 days at room temperature for sporulation. Rice seedlings were grown in a humidity-controlled greenhouse for about 3 weeks (three to four leaves) under natural conditions. Seedlings were spray inoculated with spores of $M$. oryzae isolates with a concentration of $5 \times 10^{5}$ spores per milliliter. After inoculation, seedlings were maintained in the dark for $24 \mathrm{~h}$ at $28^{\circ} \mathrm{C}$ and then, kept under high humidity for about 5 to 7 days to evaluate their symptoms.

The 0 to 5 standard scale was used to score the disease reaction as previously described (Campbell et al. 2004); briefly, "0" indicates no evidence of infection, "1" indicates brown speckling $(<1 \mathrm{~mm})$, "2" indicates brown specks ( 1 to $2 \mathrm{~mm}$ ), " 3 " indicates round to elliptical lesions ( 2 to $4 \mathrm{~mm}$ ) with gray center and brown margins, " 4 " indicates spindle-shaped lesions with necrotic centers capable of sporulation, and " 5 " indicates coalesced type 4 lesions that have killed the majority of the leaf blade. Plants with lesion scores from 0 to 2 were considered to be resistant. Plants with lesion scores 3, 4, and 5 were considered as moderately resistant, susceptible, and highly susceptible, respectively. For the field disease evaluation, varieties were grown at the station for testing blast resistance in Shanghang, Fujian, China, in 2017. Disease evaluation was carried out on leaf and spike of seeding and maturing stages, respectively.

DNA extraction. Leaf tissues from rice seedlings were collected for DNA extraction. About $100 \mathrm{mg}$ of young leaves were collected in Eppendorf tubes and frozen in liquid nitrogen. The samples were ground into fine powder with a TissueLyser (Qiagen, Haan, Germany), and genomic DNA was extracted from ground tissues using the cetyltrimethyl ammonium bromide method (Murray and Thompson 1980).

Marker development. The genomic sequences of the Pi2/9 locus in the rice varieties C101A51 (DQ352453), 75-1-127 (DQ285630), Toride 1 (DQ352040), Gumei4 (KU905633.2), and Nipponbare (DQ454158) were retrieved from GenBank (https://www.ncbi.nlm. nih.gov/), and they were aligned using Clustal (Larkin et al. 2007). The PCR was performed in $25 \mu \mathrm{l}$, and the mixture contained $12.5 \mu \mathrm{l}$ of $2 \times$ Mix buffer $\left(\mathrm{Mg}^{2+}\right.$ Plus), $1 \mu$ l of each primer (10 $\mathrm{mM})$, and $0.2 \mu \mathrm{l}$ Taqase $(5 \mathrm{U} / \mathrm{ml})$. Approximately 20 to $50 \mathrm{ng}$ of genomic DNA was added in a total volume of $25 \mu \mathrm{l}$ for PCR analysis. PCR was performed with the following thermal profile: initial denaturation at $94^{\circ} \mathrm{C}$ for 5 min followed by 32 cycles of denaturation at $94^{\circ} \mathrm{C}$ for $30 \mathrm{~s}$ and $60^{\circ} \mathrm{C}$ for $30 \mathrm{~s}$ and extension at $72^{\circ} \mathrm{C}$ for $30 \mathrm{~s}$ and a final extension at $72^{\circ} \mathrm{C}$ for $10 \mathrm{~min}$. The PCR products were separated by $3 \%$ agarose gels in $1 \times$ Tris-acetate-EDTA (TAE) electrophoresis buffer. Electrophoresis was carried out at 150 to $200 \mathrm{~mA}$ for $45 \mathrm{~min}$, and images were taken using a FluorChem SP gel imaging system (Alpha Innotech, San Leandro, CA).

Validation of the Pigm/2/9InDel linkage to the $R$ genes in $\mathbf{F}_{2}$ populations. The cultivars GufengB (Pigm) (Deng et al. 2017), Hui316 (susceptible), and Minghui86 (Piz-t) (Tian et al. 2016) maintained at the Fujian Provincial Key Laboratory of Genetic Engineering for Agriculture, Fujian Academy of Agricultural Sciences were used to make crosses with Nipponbare (susceptible), 75-1-127 (Pi9), and Nipponbare, respectively, and develop $F_{2}$ populations. The $F_{2}$ populations were genotyped with the Pim/2/9InDel. The segregations of the $R$ genes in the $\mathrm{F}_{2}$ populations indicated by the Pigm/2/ 9InDel marker are shown in Supplementary Fig. S1.

\section{Results}

Development and validation of marker Pigm/2/9InDel. We previously developed the InDel marker Pi9-Pro, which can differentiate three different genotypes corresponding to the Pi9, Pi2/Piz-t, and non-Pi2/Piz-t/Pi9 alleles (Tian et al. 2016). However, when Pi9Pro was subjected to identify the Pigm allele (Gumei 4), it produced an amplified fragment with similar size of the fragments from nonfunctional alleles (Nipponbare and 9311) (Fig. 1A). Therefore, Pi9Pro is not effective in differentiating the Pigm allele. To develop an efficient DNA marker for distinguishing the functional alleles at the Pi2/9 locus, we first analyzed genomic sequences of this locus in Gumei 4 (carrying Pigm), 75-1-127 (carrying Pi9), C101A51 (carrying $P i 2$ ), and Toride 1 (carrying $P i z-t$ ) (Fig. 1B). A primer pair, called Pigm/2/9InDel, was designed to target three positions at the Pigm locus, which produced a 164-bp amplified fragment within Pigm- $\mathrm{R}$ and $\mathrm{Nbs} 4$ tandem repeat and a 132-bp fragment at the downstream of $N b s 2$ (Fig. 1C). When Pigm/2/9InDel was used to amplify $\mathrm{Pi}$, Pi2, and Piz- $t$ donors, only one 132-bp band was amplified at the downstream of $\mathrm{Pi} 9(\mathrm{Nbs} 2)$ and $\mathrm{Nbs} 5$ tandem repeat in 75-1-127; two PCR amplicons with similar sizes of 166 and $165 \mathrm{bp}$ were produced within Pi2/Piz-t (Nbs4) and the Nbs2 tandem repeat, respectively, in both C101A51 and Toride 1 (Fig. 1C and D). For the nonfunctional alleles from Nipponbare and 9311, Pigm/2/9InDel produced bands with similar sizes of $>1,000 \mathrm{bp}$.

To validate the linkage of the Pigm/2/9InDel marker with Pigm, $\mathrm{Pi}$, and Pi2/Piz-t alleles, 168, 144, and 140 plants were randomly selected from the $\mathrm{F}_{2}$ populations derived from crosses of GufengB (Pigm) with Nipponbare, 75-1-127 (Pi9) with Hui316, and Minghui86 $(P i z-t)$ with Nipponbare, respectively. The genotype analysis revealed that the ratios of Pigm-, Pi9-, and Piz-t-containing plants to non-Pigm/Pi9/Piz-t alleles-containing plants were 121: $47\left(\chi^{2}=0.536\right), 111: 33\left(\chi^{2}=0.679\right)$, and 100:40 $\left(\chi^{2}=0.500\right)$, respectively, all of which fit the 3:1 ratio (Supplementary Fig. S1). These results indicated that Pigm/2/9InDel is effective in differentiating the Pigm, Pi9, Pi2/Piz-t and nonfunctional alleles at the Pi2/9 locus.

Distribution of functional Pi2/9 alleles in the varieties from major rice-producing areas of China. We have developed the cleaved amplified polymorphic sequence marker Pi2-LRR to differentiate the Pi2 allele from the non-Pi2 alleles (Tian et al. 2016). Therefore, Pigm/2/9InDel and Pi2-LRR were used in combination to investigate the distribution of the functional Pi2/9 alleles in Chinese rice varieties. A set of 905 rice varieties included 681 collected from the major rice-producing areas in China, among which 195 were from Guangdong, 151 were from Fujian, 74 were from Zhejiang, 73 were from Jiangsu, 36 were from Hunan, 34 were from Sichuan, 22 were from Yunnan, 19 were from Guangxi, and 77 were from other provinces of China; 119 were from the IRRI; and 105 were from other or unknown areas (Supplementary Table S1). These materials were genotyped with the Pigm/2/9InDel and Pi2-LRR markers to identify the resistance alleles of the Pi2/9 locus (partial results were shown in Fig. 2A and C). The results indicated that, among 
the tested varieties, 9, 62, and 60 had the Pigm, Pi2, and Piz-t functional alleles, respectively (Table 1). To confirm the results by Pigm/ 2/9InDel, the Pi9-Pro marker was used to determine the genotypes of the above functional alleles-containing varieties. The presence of Pi9 or Pi2/Piz- $t$ alleles identified by Pi9-Pro was consistent with that identified by Pigm/2/9InDel (Fig. 2B). Furthermore, all of the varieties carrying Pigm, Pi9, Pi2, or Piz- $t$ were tested with the developed molecular markers Pigm-4, Pi9SNP, Pi2SNP, or Pizt-PA (Hua et al. 2015; Zeng et al. 2018). As shown in Supplementary Figure S2, the identification results by Pigm/2/9InDel and Pi2-LRR were consistent with those by Pigm-4, Pi9SNP, Pi2SNP, and Pizt-PA, which proves the effectiveness of Pigm/2/9InDel in identifying the functional alleles at the Pi2/9 locus.

Interestingly, it was found that the Pigm allele was carried in the varieties collected from both Fujian and Guangdong but absent in varieties from other provinces, whereas all of the 62 varieties carrying the Pi2 allele were from Guangdong (Fig. 3). The
60 varieties carrying $P i z-t$ were from various race-planting areas. $P i 9$ was only identified in IR0436-1 from the IRRI, but it was not found in any of the tested Chinese varieties (Fig. 3). Therefore, our results indicated that Pigm/2/9InDel is an efficient marker for screening rice materials with or without functional alleles of the Pi2/9 locus.

Determining resistance of the varieties carrying the functional $P i 2 / 9$ alleles against diverse regional rice blast isolates. To investigate the effectiveness of Pigm, Pi2, and Pizt alleles identified by Pigm/2/9InDel, we selected five Pi2-carrying varieties (BL123, Huazhan, Xin 1221, Xin 1223, and Guangchao 128) collected from Guangdong province, four Pigm-carrying varieties (Minbeiwanxian, Fuyi A, Guangkang 13A, and Gufeng A) from Fujian province, and three Piz-t-carrying varieties (Chenghui 707, Hang 1, and Minghui 86) to be inoculated with isolates KJ201 and CHE86061, which are avirulent to the functional alleles of the Pi2/9 locus (Zhou et al. 2006). The varieties with nonfunctional alleles (CO39, Nipponbare,
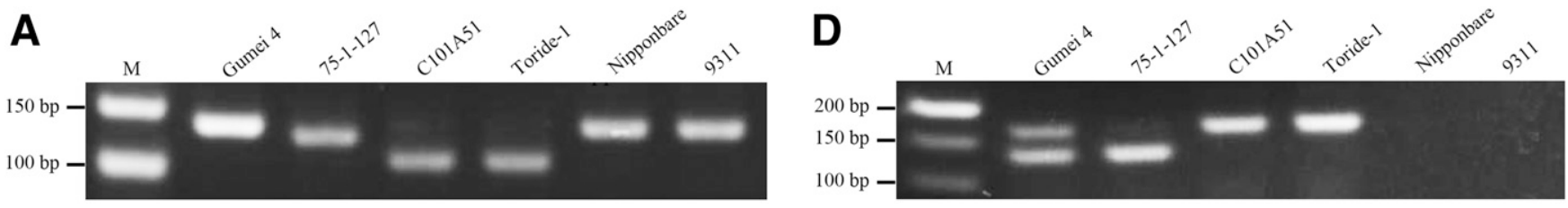

B

Pi2/z-t NPS2: $\mathrm{Pi} 2 / \mathrm{z}-\mathrm{t}$ NPS4: Pi9_NPS2: Pi9 NPS5:

Pigm_NPS2: Pigm NPS4: Pigm_NPS6:

Pi2/z-t NPS2: Pi2/z-t_NPS4: Pi9_NPS2: Pi9 NPS5: Pigm NPS2:

Pigm_NPS4:

Pigm_NPS6:

Pi2/z-t_NPS2 Pi2/z-t NPS4: Pi9 NPS2: Pi9 NPS5: Pigm_NPS2: Pigm NPS4: Pigm_NPS6:
Pigm2/9InDel-F

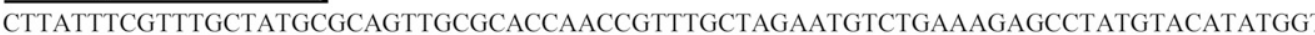
CTTATTTCGTTTGCTATGCGCAGTTGCGCACCAACCGTTTGCTAGAATGTCTGAAAGAGCCTATGTACATATGGT

1 CTTATTTCGTTTGCTATGAGCA - - - - . - CCAATCGTTTGCTAGAATGTCTGAAAGATCTTGTGTACATATGGT 1 CTTATTTCGTTTGCTATGAGCA - - - - - CCAATCGTTTGCTAGAATGTCTGAAAGATCTTGTGTACATATGGT 1 CTTATTTCGTTTGCTATGAGCA - - - - - CCAATCGTTTGCTAGAATGTCTGAAAGATCTTGTGTACATATGGT 1 CTTATTTCGTTTGCTATGCGCAGTTGCGCACCAACCGTTTGCTAGAATGTCTGAAAGAGCCTATGTACATATGGT 1 CTTATTTCGTTTGCTATGCGCAGTTGCGCACCAACCGTTTGCTAGAATGTCTGAAAGAGCCTATGTACATATGGT

76 GGCCTGAACA - - - - - . - TTACAAGTTATCATATTTTATATTGTTGCTAGCTTTCCTTTCAAAAAAAAAAAAA - TT 142 76 GGCCTGAACA - - - - - . TTACAAGTTATCATATTTTATATTGTTGCTAGCTTTCCTTTCAAAAAAAAAAAAAATT 143 68 GGACTGAACAATTGAACATTACAAGTTATCATATTTTATATT $\cdots \cdots$ 68 GGACTGAACAATTGAACATTACAAGTTATCATATTTTATATT $\cdots \ldots$

68 GGACTGAACAATTGAACATTACAAGTTATCATATTTTATATT $\cdots \cdots$ 76 GGCCTGAACA - - - - - TTACAAGTTATCATATTTTATATTGTTGCTAGCTTTCCTTTCAAAAAAAAAAAA - - TT 142 76 GGCCTGAACA - . - . - . TTACAAGTTATCATATTTTATATTGTTGCTAGCTTTCCTTTCAAAAAAAAAAAA - TT 142

C

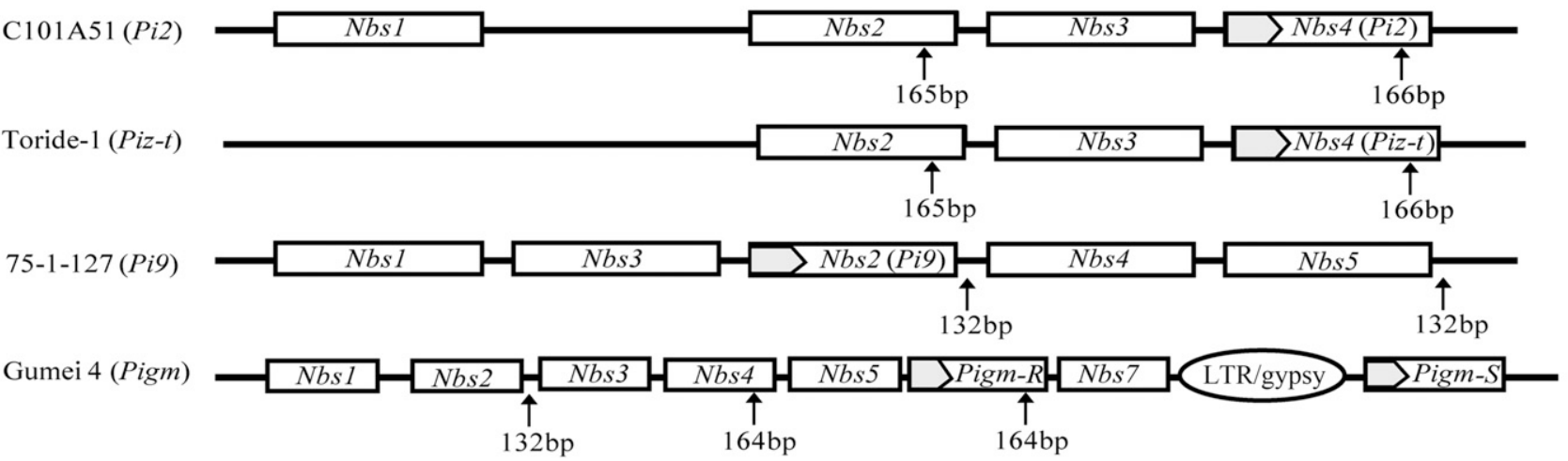

Fig. 1. Development of the insertion/deletion (InDel) marker Pigm/2/9lnDel for differentiating functional alleles at the Pi2/9 locus. A, Identification of Pigm, Pi9, Pi2, and Piz-t alleles in Gumei 4, 75-1-127, C101A51, and cultivar Toride-1, respectively, with the Pi9-Pro marker, which could not distinguish Pigm from the nonfunctional alleles in Nipponbare and 9311. B, Pigm/2/9InDel primers are designed for targeting the genomic variation of the Pi2/9 locus in Gumei 4, 75-1-127, C101A51, and Toride-1. C, Physical location of the identified InDel region by Pigm/2/9InDel and sizes of its amplicons at the Pi2/9 locus. D, Identification of the functional alleles with the Pigm/2/9InDel marker. M, DNA ladder. LTR, long terminal repeat. 
and Lijiangxintuanheigu [LTH]) were included as controls. Our results indicated that all of these identified varieties showed resistance against the two isolates, whereas Co39, Nipponbare, and LTH were susceptible (Table 2).

To further determine resistance of these functional allelescontaining varieties to regional blast isolates, we inoculated them with four Fujian isolates (FJ501-3, FJ86, RB18, and RB16) and four Guangdong isolates (CHL2110, CHL768, RB9, and RB6). In addition, evaluation of blast disease reactions in the field was also carried out by growing these varieties at the station for testing blast resistance in Shanghang, Fujian, China, in 2017. The results showed that all of the four Pigm-carrying Fujian varieties and five Pi2-carrying Guangdong varieties were highly resistant to the Fujian and Guangdong isolates, respectively (Table 2). However, variety Huazhan from Guangdong was susceptible to the Fujian isolate FJ86. Varieties Gumei 4 and Gufeng A from Fujian were moderately resistant and susceptible to Guangdong isolates RB9 and CHL2110, respectively, and variety Fuyi A displayed moderate resistance and susceptibility to Guangdong isolates RB9 and RB6, respectively (Table 2). It was also found that 75-1-127 carrying $\mathrm{Pi} 9$ was resistant to all of the Fujian and Guangdong isolates except CHL2110. Furthermore, the results of blast evaluation in the Shanghang field by natural infection indicated that all of the varieties carrying Pigm, Pi2, or Pi9 displayed high resistance except Huazhan, Fuyi A, and Gufeng A, which were moderately resistant (Table 2). However, the four varieties carrying the Piz-t allele displayed susceptibility to most Fujian and Guangdong isolates and in the field trial (Table 2), suggesting that Piz- $t$ almost lost its resistance to $M$. oryzae in these provinces. Our results suggested that different functional alleles at the Pi2/9 locus could contribute to the specific resistance to regional $M$. ory$z a e$ isolates.

\section{Discussion}

It is widely accepted that the most effective and environmentally friendly method to control blast disease is pyramiding $R$ genes into rice cultivars through breeding. However, the traditional phenotype-based rice breeding strategy is inefficient and may be inaccurate in screening the desired resistant progeny under field conditions for the reason that disease reaction and severity levels are affected by many environmental factors. Development of molecular markers linked to the desired traits can improve the efficiency and accuracy of conventional resistance breeding. With increased numbers of cloned R genes, gene-specific markers can be developed from portions of the gene itself for MAS, and MAS has already been proven to be very helpful in rice blast resistance breeding because the blastresistant phenotypes are usually controlled by a single or few genes (Srivastava et al. 2017). However, to control rice blast disease, it is also important to identify the $R$ genes that are carried by diverse cultivars and determine the effective $R$ genes against $M$. oryzae isolates.

The $R$ genes at the $P i 2 / 9$ locus, especially $P i 2, P i 9$, and Pigm, have provided rice cultivars with broad-spectrum resistance against blast disease in the main rice-producing areas (Chen et al. 2001; Deng et al. 2017; Du et al. 2015; Li et al. 2005; Yang et al. 2008). A series of markers has been developed by differentiating the sequence polymorphism of functional alleles at this locus. For example, Hua et al. (2015) developed Pi9SNP, Pi2SNP, and Pizt-PA markers by utilizing the sequence differences among Pi9, Pi2, and Piz-t, and these

Table 1. Summary of the collected varieties carrying Pigm, Pi9, Pi2, and Piz-t alleles

\begin{tabular}{ll}
\hline Alleles & \multicolumn{1}{c}{ Varieties } \\
\hline Pi2 (62) & Aihuasizhan, BL123, Guangchao 128, Fengerzhan, \\
& Fengruanzhan, Fengsizhan, Fengtaisimiao, Fengtaizhan, \\
& Fengxianzhan, Fengxiusimiao, Fengyuezhan, Guangchao \\
& 1222, Guangjinruanzhan, Guangyinzhan, Guangyuanzhan12, \\
& Gujinzhan, Helisimiao, Huahang 52, Huahangyin, \\
& Huangguangsimiao, Huangguangxinzhan, Huangguangzhan, \\
& Huangsizhan, Huangxiusimiao, Huaxiaruanzhan, Huazhan, \\
& Jinhuaruanzhan, Lifengzhan 1, Lingnansimiao, Meiyazhan, \\
& Meiyouzhan, Moliruanzhan, Molisimiao, Qifozhan, \\
& Tianjinruanzhan, Wushanfengzhan, Wushanhuadao, \\
& Wushanlizhan, Wushanyouzhan, Wuyuezhan 2, Xin 1221, \\
& Xin 1223, Xiaolixiangzhan, Xinsimiao, Xinyuanzhan, \\
& Yinfengyouzhan, Youzhan 13, Yefengzhan, Yueguangsimiao, \\
& YueJinyouzhan, Yuekang 1221, Yuekang 1222, Yuekang \\
& 1223, Yuekang 1224, Yuenongsimiao, Yuetaiyouzhan, \\
& Yueyinzhan, Yueyouxinzhan, Yuezhenzhan, Yushansizhan, \\
Zaonantonggu, Zhongguangsimiao 5. \\
Haobuka, Haomake(K), Jiabala, Jiazao 935, Fengxinzhan, Hang \\
1hao, Guisixiangnuo, Duohui 43, Minghui 86, Zhi 07, Keyou \\
80, Zhi 05W, Hui 68, Shuiyuan 290, Liaodong 218, FSJ010, \\
R1259, GIZA 176, Jiazao 1hao, Jiahua 1, Xiushui09, Xiushui \\
128, Xiushui 110, Chunjiang 03, Chunjiang 101, Chunjiang \\
104, Jia 66, Xiushui 33, Xianghu30, Zhejing 41, Yongnuo 34, \\
Ning 88, Xiushui 134, Xiushui 321, Xiushui 05, Xiushui 03, \\
Chunjiang 110, Tai 400, Jia 58, Xiushui 123, Jia 203, \\
Qifanzhan, Guisixiangnuo, IR0429-1, IR0432-1, Gangannuo, \\
IR0434, IR0435, IR0445, IR0446, IR04554, IR04555, \\
IR04556, IR04557, IR0467, IR4774-295-2-3, IR73712-68-3-1- \\
2, IR72164-368-6-2-2-2, IR1317, IR59552-63-2-3. \\
Anfeng A, Fuyi A, Guangkang 13A, Meibeiwanxian, \\
Fengxiangsimiao 2, Hefengsimiao, Lifengzhan, \\
Yuefengxinzhan, Yuetaizaozhan. \\
Piz-t (60)-1
\end{tabular}
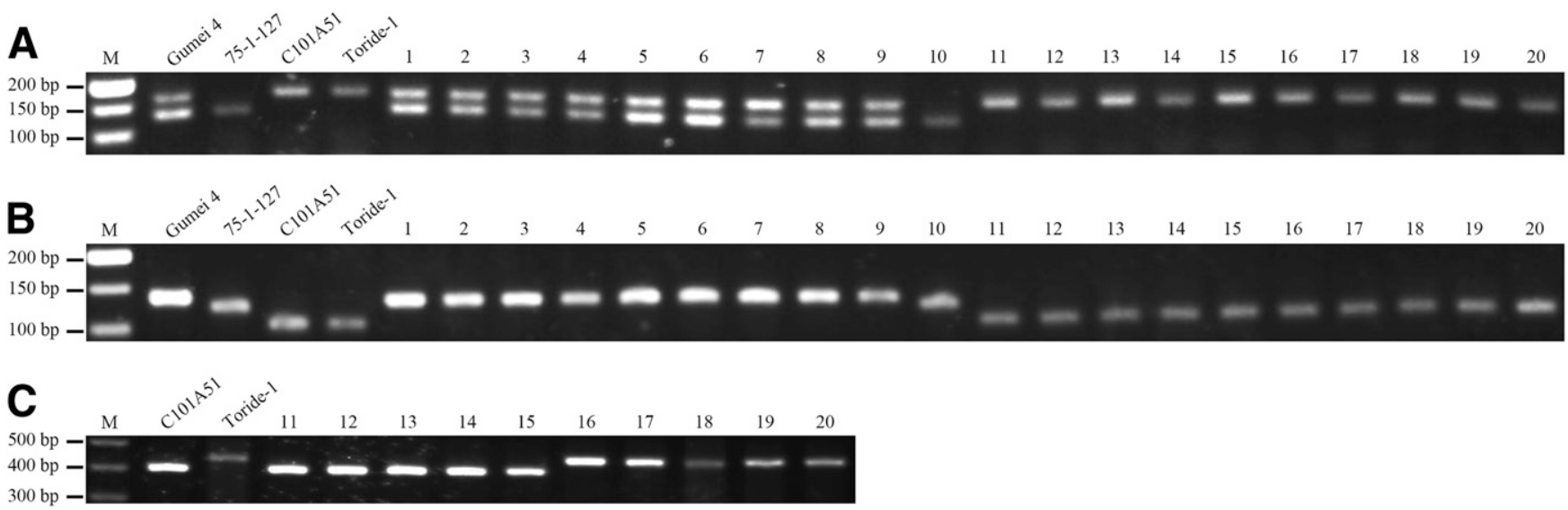

Fig. 2. Identification of the functional alleles at the Pi2/9 locus in collected varieties with the insertion/deletion (InDel) marker Pigm/2/9InDel, the Pi9-Pro marker, and the Pi2-LRR marker. The PCR amplification patterns with A, Pigm/2/9lnDel, B, Pi9-Pro, and C, Pi2-LRR. The analyzed varieties are as follows: 1, Anfeng A; 2, Fengxiangsimiao 2; 3, Fuyi A; 4, Guangkang 13A; 5, Hefengsimiao; 6, Lifengzhan; 7, Meibeiwanxian; 8, Yuefengxinzhan; 9, Yuetaizaozhan; 10, IR0436-1; 11, Aihuasizhan; 12, Guangchao 128; 13, Fengerzhan; 14, Fengruanzhan; 15, Fengsizhan; 16, Haobuka; 17, Haomake (K); 18, Jiabala; 19, Jiazao 935; 20, Fengxinzhan. M, DNA ladder. 
markers were used to determine the presence and absence of the $R$ genes in 101 rice varieties. In 2019, Wang et al. (2019) designed the marker GMR-3 to identify the PigmR variant in rice varieties. However, none of these markers can individually distinguish multiple functional alleles. Although Pi9-Pro is able to differentiate the $\mathrm{Pi}$, Pi2/Piz-t, and non-Pi2/Piz-t/Pi9 alleles, it is not effective in distinguishing the cloned Pigm from the nonfunctional alleles. Pigm/2/ 9InDel developed in this study not only differentiates Pigm, $\mathrm{Pi}$, $\mathrm{Pi}$ / $P i z-t$, and the nonfunctional alleles but also, separates the target alleles in $\mathrm{F}_{2}$ populations, making it more efficient in MAS for varieties with the resistance alleles at the Pi2/9 locus.

Among the 905 germplasms tested in this study, all 62 varieties carrying Pi2 were collected from Guangdong province, and 9 varieties carrying Pigm were collected from both Fujian and Guangdong provinces. No Chinese variety contains the $P i 9$ allele. In contrast, varieties from a wide range of rice-producing areas carry Piz-t. Most of the tested varieties carrying $P i 2$ displayed high resistance to Fujian isolates and in the field trial, whereas this allele was found in few Fujian varieties, suggesting that $P i 2$ is a potential resistance resource to be utilized in Fujian province. However, nearly all of the varieties from Guangdong that carry the Pi2 or Pigm alleles were developed in recent years, implying that these two $R$ genes have been intentionally used in the breeding programs in this area.

Previous studies showed that $\mathrm{Pi} 2$ and $\mathrm{Pi}$ conferred high resistance against rice blast isolates from Guangdong, Fujian, Guizhou, and Hunan (Pan et al. 2008; Yang et al. 2008; Zhu et al. 2004). However, Pi2 has become less effective after cultivars with the gene have grown over the years in Guangdong along with the evolution of M. oryzae population (Ling et al. 1989; Wang et al. 2012; Yang et al. 2008; Zhu et al. 2004, 2008). Likewise, Piz- $t$ was first used to develop cultivars resistant to rice blast in the 1970s, and the germplasms carrying Piz- $t$ have gradually lost their resistance in many rice-producing areas (Lei et al. 2000; Yang et al. 2014). However, $\mathrm{Pi} 9$ and $\mathrm{Pigm}$ have still shown broad-spectrum resistance to rice blast (Wu et al. 2015; Yang et al. 2008; Yu et al. 2013; Yuan et al. 2017), which is consistent with our results. Considering that $P i 9$ is not widely present in Chinese rice varieties, it could be a valuable resistance resource for use in China. In addition, our results suggested that Pi2 and Pigm are not widely distributed in the varieties grown in provinces other than Fujian and Guangdong. Therefore, these resistance alleles are still tenable for large-scale rice resistance breeding in China (Wang et al. 2017).

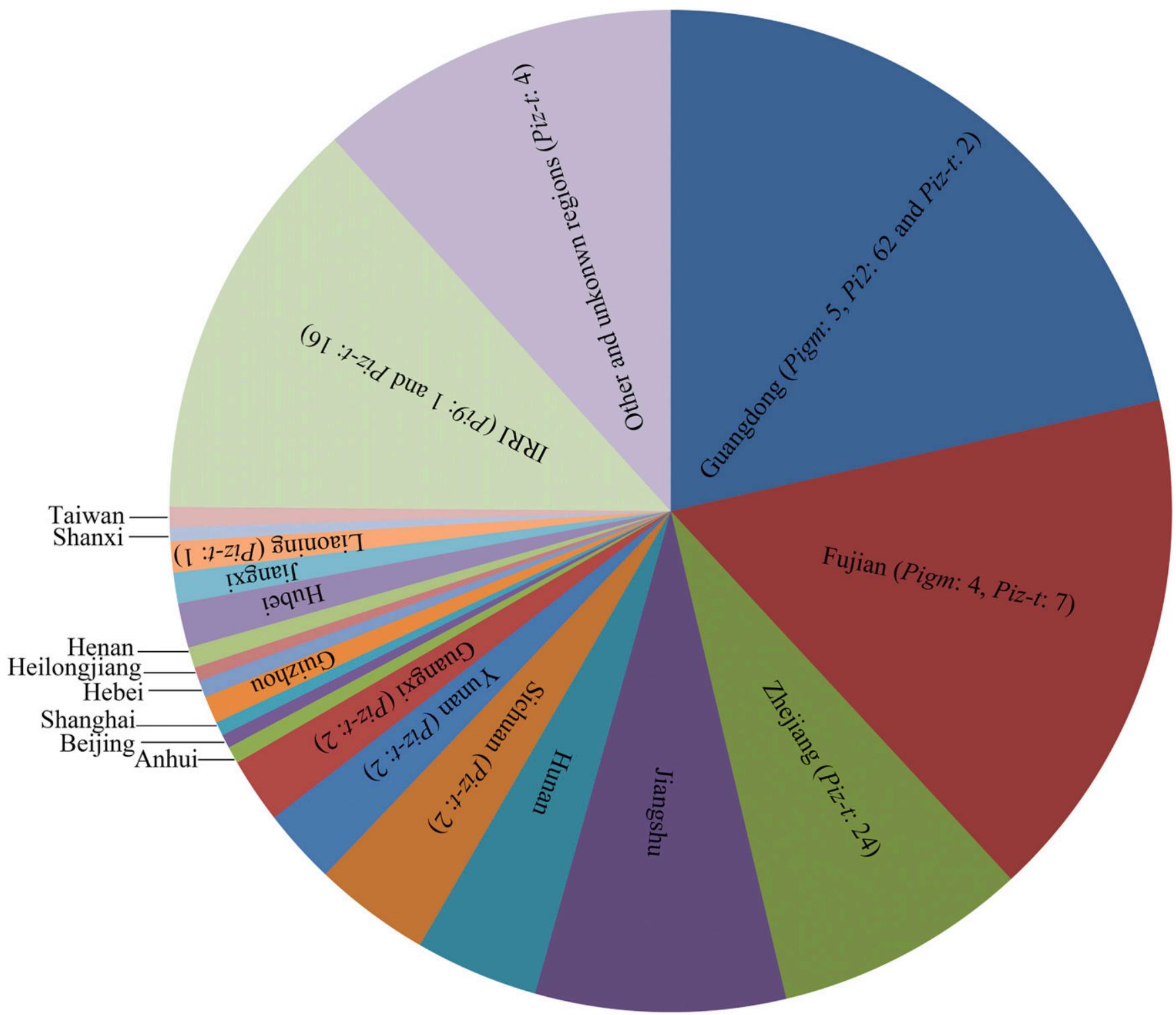

Fig. 3. Regional distribution of all of the varieties collected in this study. The numbers of Pigm-, Pi9-, Pi2-, and Piz-t-containing varieties are shown on the pie chart. IRRI, International Rice Research Institute. 
Table 2. Disease incidence of the varieties containing Pigm, Pi9, Pi2, or Piz-t alleles inoculated with various isolates of Magnaporthe oryzae and in the field disease trial

\begin{tabular}{|c|c|c|c|c|c|c|c|c|c|c|c|c|}
\hline \multirow[b]{3}{*}{ Gene } & \multirow[b]{3}{*}{ Cultivar } & \multirow{2}{*}{\multicolumn{2}{|c|}{ Avirulent strains }} & \multirow{2}{*}{\multicolumn{4}{|c|}{ Guangdong isolates }} & \multicolumn{5}{|c|}{ Fujian isolates } \\
\hline & & & & & & & & \multirow[b]{2}{*}{ RB18 } & \multirow[b]{2}{*}{ RB16 } & \multirow[b]{2}{*}{ FJ501-3 } & \multirow[b]{2}{*}{ FJ86 } & \multirow{2}{*}{$\begin{array}{c}\text { Shanghang } \\
\text { (2017) }\end{array}$} \\
\hline & & KJ201 & CHE86061 & RB9 & RB6 & CHL2110 & CHL768 & & & & & \\
\hline \multirow[t]{6}{*}{$\mathrm{Pi2}$} & C101A51 & 0 & 0 & 0 & 0 & 0 & 0 & 0 & 0 & 0 & 0 & 0 \\
\hline & BL123 & 0 & 0 & 0 & 0 & 0 & 0 & 0 & 0 & 0 & 0 & 0 \\
\hline & Huazhan & 0 & 0 & 0 & 0 & 0 & 0 & 0 & 0 & 0 & 4 & 3 \\
\hline & Xin 1221 & 0 & 0 & 0 & 0 & 0 & 0 & 0 & 0 & 2 & 0 & 0 \\
\hline & Xin 1223 & 0 & 0 & 0 & 0 & 0 & 0 & 0 & 0 & 0 & 0 & 0 \\
\hline & Guangchao 128 & 0 & 0 & 0 & 1 & 0 & 0 & 0 & 0 & 0 & 0 & 0 \\
\hline \multirow[t]{5}{*}{ Pigm } & Gumei 4 & 0 & 0 & 3 & 0 & 0 & 0 & 0 & 0 & 0 & 0 & 0 \\
\hline & Minbeiwanxian & 0 & 0 & 0 & 0 & 0 & 0 & 0 & 0 & 0 & 0 & 0 \\
\hline & Fuyi A & 0 & 0 & 3 & 4 & 0 & 0 & 0 & 0 & 0 & 0 & 3 \\
\hline & Guangkang 13A & 0 & 0 & 2 & 0 & 0 & 0 & 0 & 0 & 0 & 0 & 0 \\
\hline & Gufeng A & 0 & 0 & 0 & 0 & 4 & 0 & 0 & 0 & 0 & 0 & 3 \\
\hline $\operatorname{PiO}$ & $75-1-127$ & 0 & 0 & 0 & 0 & 4 & 0 & 0 & 2 & 0 & 0 & 0 \\
\hline \multirow[t]{4}{*}{ Piz-t } & IRBLzt-T & 0 & 0 & 3 & 1 & 4 & 0 & 5 & 4 & 5 & 5 & 5 \\
\hline & Chenghui 707 & 0 & 0 & 0 & 2 & 0 & 0 & 1 & 2 & 1 & 3 & 3 \\
\hline & Hang 1 & 0 & 0 & 1 & 3 & 4 & 4 & 2 & 4 & 3 & 5 & 4 \\
\hline & Minghui86 & 2 & 1 & 2 & 3 & 0 & 0 & 1 & 4 & 4 & 5 & 5 \\
\hline \multirow{3}{*}{$\begin{array}{l}\text { Non-functional } \\
\text { alleles }\end{array}$} & Co39 & 5 & 5 & 4 & 4 & 4 & 5 & 5 & 5 & 5 & 5 & 5 \\
\hline & Nipponbare & 4 & 4 & 5 & 5 & 3 & 5 & 5 & 5 & 5 & 5 & 5 \\
\hline & LTH & 5 & 5 & 5 & 5 & 3 & 5 & 5 & 5 & 5 & 5 & 5 \\
\hline
\end{tabular}

\section{Literature Cited}

Bryan, G. T., Wu, K.-S., Farrall, L., Jia, Y., Hershey, H. P., McAdams, S. A., Faulk, K. N., Donaldson, G. K., Tarchini, R., and Valent, B. 2000. A single amino acid difference distinguishes resistant and susceptible alleles of the rice blast resistance gene Pi-ta. Plant Cell 12:2033-2045.

Campbell, M. A., Chen, D., and Ronald, P. C. 2004. Development of co-dominant amplified polymorphic sequence markers in rice that flank the Magnaporthe grisea resistance gene $\mathrm{Pi}(\mathrm{t})$ in recombinant inbred line 29. Phytopathology 94:302-307.

Chen, D. H., Zeigler, R. S., Ahn, S. W., and Nelson, R. J. 1996. Phenotypic characterization of the rice blast resistance gene $P i-2(t)$. Plant Dis. 80:52-56.

Chen, H. L., Chen, B. T., Zhang, D. P., Xie, Y. F., and Zhang, Q. F. 2001. Pathotypes of Pyricularia grisea in rice fields of central and southern China. Plant Dis. 85:843-850.

Chen, Z., Zheng, Y., Wu, W., and Zhao, C. 2004. Screening and application of an SSR marker closely linked to $P i-2(t)$, a gene resistant to rice blast. Mol. Plant Breed. 2:321-325.

Deng, Y., Zhai, K., Xie, Z., Yang, D., Zhu, X., Liu, J., Wang, X., Qin, P., Yang, Y., Zhang, G., Li, Q., Zhang, J., Wu, S., Milazzo, J., Mao, B., Wang, E., Xie, H., Tharreau, D., and He, Z. 2017. Epigenetic regulation of antagonistic receptors confers rice blast resistance with yield balance. Science 355:962-965.

Dodds, P. N., and Rathjen, J. P. 2010. Plant immunity: Towards an integrated view of plant-pathogen interactions. Nat. Rev. Genet. 11:539-548.

Du, T., Yu, X., Zhu, S., Li, G., and Ding, J. 2015. Resistance evaluation of polymeric rice material with blast resistance genes $P i 1$ and $P i 9$. Guizhou Agric. Sci. 43:35-38.

Fu, C., Wu, T., Liu, W., Wang, F., Li, J., Zhu, X., Huang, H., Zhen, R. L., Liao, Y., Zhu, M., Chen, J., and Huang, Y. 2012. Genetic improvement of resistance to blast and bacterial blight of the elite maintainer line Rongfeng B in hybrid rice (Oryza sativa L.) by using marker-assisted selection. Afr. J. Biotechnol. 11: 13104-13124.

Hayashi, K., Yoshida, H., and Ashikawa, I. 2006. Development of PCR-based allele-specific and InDel marker sets for nine rice blast resistance genes. Theor. Appl. Genet. 113:251-260.

Hua, L. X., Wang, W. J., Chen, S., Wang, C. Y., Zeng, L. X., Yang, J. Y., Zhu, X. Y., and Jing, S. U. 2015. Development of specific DNA markers for detecting the rice blast resistance gene alleles Pi2/9/z-t. Chin. J. Rice Sci. 32:453-461.

Jia, Y., Redus, M., Wang, Z., and Rutger, J. N. 2004. Development of a SNLP marker from the Pi-ta blast resistance gene by tri-primer PCR. Euphytica 138:97-105

Jia, Y., Wang, Z., and Singh, P. 2002. Development of dominant rice blast Pi-ta resistance gene markers. Crop Sci. 42:2145-2149.

Jiang, J., Mou, T., Yu, H., and Zhou, F. 2015. Molecular breeding of thermosensitive genic male sterile (TGMS) lines of rice for blast resistance using the $P i 2$ gene. Rice (N. Y.) 8:11.

Jiang, N., Li, Z., Wu, J., Wang, Y., Wu, L., Wang, S., Wang, D., Wen, T., Liang, Y., Sun, P., Liu, J., Dai, L., Wang, Z., Wang, C., Luo, M., Liu, X., and Wang, G. 2012a. Molecular mapping of the Pi2/9 allelic gene Pi2-2 conferring broadspectrum resistance to Magnaporthe oryzae in the rice cultivar Jefferson. Rice (N. Y.) 5:29.
Jiang, N., Wang, S. H., Li, Z.-Q., Wen, T., Liang, Y., Wu, J., Dai, L. Y., Wang, G. L., and Liu, X. L. 2012b. Analysis of the antimicrobial spectrum of three rice blast resistance genes at Pi2/9 locus and genetic diversity of rice blast strains. J. Hunan Agric. Univ. 38:506-510.

Khanna, A., Sharma, V., Ellur, R. K., Shikari, A. B., Krishnan, S. G., Singh, U. D., Prakash, G., Sharma, T. R., Rathour, R., and Variar, M. 2015. Development and evaluation of near-isogenic lines for major blast resistance gene(s) in Basmati rice. Theor. Appl. Genet. 128:1243-1259.

Koide, Y., Kawasaki, A., Telebanco-Yanoria, M. J., Hairmansis, A., Nguyet, N. T. M., Bigirimana, J., Fujita, D., Kobayashi, N., and Fukuta, Y. 2010. Development of pyramided lines with two resistance genes, Pish and Pib, for blast disease (Magnaporthe oryzae B. Couch) in rice (Oryza sativa L.). Plant Breed. 129:670-675.

Larkin, M., Blackshields, G., Brown, N. P., Chenna, R., Mcgettigan, P. A., McWilliam, H., Valentin, F., Wallace, I. M., Wilm, A., Lopez, R., Thompson, J. D., Gibson, T. J., and Higgins, D. G. 2007. Clustal W and clustal X version 2.0. Bioinformatics 23:2947-2948.

Lei, C., Ling, Z., Wang, J., and Jiang, W. 2000. Variation of physiological races of blast fungus in northern rice growing region of China and resistance breeding strategies. Crop J. 3:14-16.

Li, J., Li, C., Chen, Y., Lei, C., and Ling, Z. 2005. Evaluation of twenty-two blast resistance genes in Yunnan using monogenetic rice lines. J. Plant Protect. 32:113-119.

Ling, Z., Wang, J., and Li, M. 1989. Studies on pathogenic races of Pyricularia oryzae in cropping rice regions of North China. Sci. Agric. Sin. 22:7-13.

Liu, G., Lu, G., Zeng, L., and Wang, G. L. 2002. Two broad-spectrum blast resistance genes, $P i 9(t)$ and $P i 2(t)$, are physically linked on rice chromosome 6. Mol. Genet. Genomics 267:472-480.

Liu, W., Liu, J., Ning, Y., Ding, B., Wang, X., Wang, Z., and Wang, G.-L. 2013. Recent progress in understanding PAMP- and effector-triggered immunity against the rice blast fungus Magnaporthe oryzae. Mol. Plant 6:605-620.

Liu, Y., Xu, P.-Z., Zhang, H.-Y., Xu, J.-D., Wu, F.-Q., and Wu, X.-J. 2008. Marker-assisted selection and application of blast resistant gene $\mathrm{Pib}$ in rice Sci. Agric. Sin. 41:9-14.

Mackill, D. J., and Bonman, J. M. 1992. Inheritance of blast resistance in nearisogenic lines of rice. Phytopathology 82:746-749.

Murray, M. G., and Thompson, W. F. 1980. Rapid isolation of high molecular weight plant DNA. Nucleic Acids Res. 8:4321-4326.

Pan, S. J., Dai, L. Y., Liu, X. L., Wu, J., Ning, Y. S., Hu, Y., Liu, J. L., and Wang, G. L. 2008. Inheritance analysis and identification of rice blast resistance of Pi9 transgenic plants in indica rice 1701. J. Hunan Agric. Univ. 34:579-582.

Qu, S., Liu, G., Zhou, B., Bellizzi, M., Zeng, L., Dai, L., Han, B., and Wang, G. L. 2006. The broad-spectrum blast resistance gene $P i 9$ encodes a nucleotidebinding site-leucine-rich repeat protein and is a member of a multigene family in rice. Genetics 172:1901-1914.

RoyChowdhury, M., Jia, Y., Jia, M. H., Fjellstrom, R., and Cartwright, R. D. 2012. Identification of the rice blast resistance gene $P i b$ in the national small grains collection. Phytopathology 102:700-706.

Scheuermann, K. K., and Jia, Y. 2016. Identification of a Pi9-containing rice germplasm with a newly developed robust marker. Phytopathology 106:871-876.

Srivastava, D., Shamim, M., Kumar, M., Mishra, A., Pandey, P., Kumar, D., Yadav, P., Siddiqui, M. H., and Singh, K. N. 2017. Current status of 
conventional and molecular interventions for blast resistance in rice. Rice Sci. 24:299-321.

Tian, D., Chen, Z., Chen, Z., Zhou, Y., Wang, Z., Feng, W., and Chen, S. 2016. Allele-specific marker-based assessment revealed that the rice blast resistance genes $P i 2$ and $P i 9$ have not been widely deployed in Chinese indica rice cultivars. Rice (N. Y.) 9:19.

Wang, F., Chen, Z., Xu, Y., Wang, J., Li, W., Fan, F., Chen, L., Tao, Y., Zhong, W., and Yang, J. 2019. Development and application of the functional marker for the broad-spectrum blast resistance gene PigmR in rice. Sci. Agric. Sin. 52:955-967.

Wang, W., Su, J., Zhang, J., Li, Y., Chen, S., Zeng, L., Yang, J., and Zhu, X. 2012. Pathogenicity analysis of the rice blast fungus isolated from the blast panicles of Yuejingsimiao 2. Guangdong Agric. Sci. 23:59-61.

Wang, W., Zhou, J., Wang, C., Su, J., Feng, J., Chen, B., Feng, A., Yang, J., Chen, S., and Zhu, X. 2017. Distribution of eight rice blast resistance genes in indica hybrid rice in China. Chin. J. Rice Sci. 31:299-306.

Wang, Z., Jia, Y., Rutger, J. N., and Xia, Y. 2007. Rapid survey for presence of a blast resistance gene $\mathrm{Pi}$-ta in rice cultivars using the dominant DNA markers derived from portions of the Pi-ta gene. Plant Breed. 126:36-42.

Wang, Z.-X., Yano, M., Yamanouchi, U., Iwamoto, M., Monna, L., Hayasaka, H., Katayose, Y., and Sasaki, T. 1999. The Pib gene for rice blast resistance belongs to the nucleotide binding and leucine-rich repeat class of plant disease resistance genes. Plant J. 19:55-64.

Wu, J. L., Fan, Y. Y., Li, D. B., Zheng, K. L., Leung, H., and Zhuang, J. Y. 2005. Genetic control of rice blast resistance in the durably resistant cultivar Gumei 2 against multiple isolates. Theor. Appl. Genet. 111:50-56.

Wu, Y., Xiao, N., Yu, L., Pan, C., Li, Y., Zhang, X., Liu, G., Dai, Z., Pan, X., and $\mathrm{Li}$, A. 2015. Combination patterns of major R genes determine the level of resistance to the M. oryzae in rice (Oryza sativa L.). PLoS One 10:e0126130.

Yang, J., Chen, S., Zeng, L., Li, Y., Chen, Z., and Zhu, X. 2008. Evaluation on resistance of major rice blast resistance genes to Magnaporthe grisea isolates collected from indica rice in Guangdong province, China. Chin. J. Rice Sci. 22:190-196.
Yang, X.-L., Zeng, F.-S., Zhang, S., and Yu, D.-Z. 2014. Analyses of twenty blast resistance genes in Hubei province. Hubei Agric. Sci. 53:3798-3802.

Yin, D. S., Xia, M. Y., Li, J. B., Wan, B. L., Zha, Z. P., Du, X. S., and Qi, H.-X 2011. Development of STS marker linked to rice blast resistance gene Pi9 in marker-assisted selection breeding. Chin. J. Rice Sci. 1:25-30.

Yu, M., Dai, Z., Pan, C., Chen, X., Yu, L., Zhang, X., Li, Y., Xiao, N., Gong, H. Sheng, S., Pan, X., Zhang, H., and Li, A. 2013. Resistance spectrum difference between two broad-spectrum blast resistance genes,Pigm and Pi2, and their interaction effect on Pil. Acta Agron. Sin. 39:1927-1934.

Yuan, X., Li, D.-Y., and Song, F.-M. 2017. Rice broad-spectrum resistance against blast disease: Molecular mechanism and applications. Plant Physiol. J. 53: 1348-1358.

Zeng, S. Y., Li, C., Du, C. C., Sun, L. T., Jing, D. D., Lin, T. Z., Yu, B., Qian, H. F., Yao, W. C., Zhou, Y. W., and Gong, H. B. 2018. Development of specific markers for Pigm in marker-assisted breeding of panicle blast resistant japonica rice. Chin. J. Rice Sci. 32:453-461.

Zhang, Y., Zhang, X.-J., Yang, F.-J., and Feng, Z.-F. 2013. SNP-based detecting method of rice blast resistance gene Pi-ta. Chin. J. Rice Sci. 27:325-328.

Zhou, B., Qu, S., Liu, G., Dolan, M., Sakai, H., Lu, G., Bellizzi, M., and Wang, G. L. 2006. The eight amino-acid differences within three leucine-rich repeats between Pi2 and Piz-t resistance proteins determine the resistance specificity to Magnaporthe grisea. Mol. Plant-Microbe Interact. 19:1216-1228.

Zhu, X., Chen, S., Yang, J., Zhou, S., Zeng, L., Han, J., Su, J., Wang, L., and Pan, Q. 2012. The identification of $P i 5 O(t)$, a new member of the rice blast resistance Pi2/Pi9 multigene family. Theor. Appl. Genet. 124:1295-1304.

Zhu, X., Yang, J., Chen, Y., Yang, W., Chen, X., Zeng, L., and Chen, S. 2008. Race identification and pathogenicity test of the blast fungus causing the resistance breakdown of hybrid rice Tianyou 998. Guangdong Agric. Sci. 12: 84-86.

Zhu, X., Yang, Q., Yang, J., Lei, C., Wang, J., and Ling, Z. 2004. Differentiation ability of monogenic lines to Magnaporthe grisea in indica rice. Acta Phytopathol. Sin. 34:361-368. 